\title{
Esophageal Involvement in Microscopic Polyangiitis: A Case Report and Review of Literature
}

\author{
Masataka Matsumoto ${ }^{1,2}$, Takefumi Nakamura ${ }^{1}$, Tsuyoshi Ohashi ${ }^{1,3}$, Tomoko Okuno ${ }^{4,5}$, \\ Kosho Takasu ${ }^{5}$, Shouichi Hoshino ${ }^{1}$, Yasushi Sugiura ${ }^{1}$, Dan Ueshima ${ }^{1}$, Naoyuki Suzuki ${ }^{1}$, \\ Suguru Uose ${ }^{1}$, Takayuki Nada ${ }^{1}$ and Kiyotaka Kawaguchi ${ }^{1}$
}

\begin{abstract}
A 72-year-old man with cough and sputum showed esophageal wall thickening and pneumonia in chest computed tomography (CT) scan. Following endoscopy, we diagnosed reflux esophagitis and subscribed proton pump inhibitor. The esophageal lesion, however, was intractable. We diagnosed microscopic polyangiitis (MPA) because of vasculitis symptoms, cytoplasmic antineutrophil cytoplasmic antibodies (cANCA) in blood and no granulomatous change in the esophagus. We adopted pulse therapy of cyclophosphamide and oral prednisolone; the symptoms and esophageal lesion were markedly improved. We concluded that the esophageal lesion was an aspect of MPA. To our knowledge, this is the first report of esophageal involvement in MPA.
\end{abstract}

Key words: microscopic polyangiitis, Wegener's granulomatosis, esophagitis, vasculitis, ANCA

(DOI: 10.2169/internalmedicine.46.6115)

\section{Introduction}

Microscopic polyangiitis (MPA) is a vasculitis syndrome similar to Wegener's granulomatosis (WG). MPA, as with WG, shows arteritis in the medium to small arteries and microangiitis especially in the lung and kidney (1). There has been no case report of MPA complicated with an esophageal lesion, though four cases of WG accompanied by esophageal lesion have been reported. Here, we report a case of MPA complicated with an esophageal lesion, which was remarkably improved by prednisolone (PSL) and cyclophosphamide (CPA).

\section{Case Report}

A 72-year-old man consulted the open clinic of the Department of Respiratory Medicine of Kitano Hospital in April 2002 complaining of cough, choking on food, bloody sputum and $3 \mathrm{~kg}$ body weight loss in one month. He had a history of Type 2 diabetes mellitus (Type 2 DM) from 66 years old, and reumatoid arthritis (RA) from 57 years old. He had chronically taken glibenclamide for Type 2 DM from the age of 60 years old. But before admission in August 2003, he had not taken non-steroidal anti-inflammatory drugs (NSAIDs) or bisphosphonates. He was a smoker of 70 cigarettes a day from 17 through 65 years old. He showed elevated C-reactive protein (CRP; $6.05 \mathrm{mg} / \mathrm{dl}$ ) and blood sugar $(148 \mathrm{mg} / \mathrm{dl})$, but his peripheral blood leucocyte count was within normal range at $8,000 / \mu$. Chest CT showed infiltrative areas in both lungs and also revealed distal esophageal wall thickening (Fig. 1).

At first, we began antitussive and some antibiotics on the diagnosis of pneumonia: Amoxicillin/Clavulanic acid (AMPC/CVA)+Clarithromycin (CAM) from April 3 to April 9, Tosufloxacin (TFLX) from April 10 to April 30, CAM from May 1 to May 21 and CAM+TFLX from May 22 to June 12. In June 2002, original pneumonia improved on chest $\mathrm{X}$ ray after a long time. However, chest CT in July 2002 revealed a new pneumonia lesion in other site. We

\footnotetext{
${ }^{1}$ Department of Gastroenterology \& Hepatology, Kitano Hospital, Osaka, ${ }^{2}$ Department of Respiratory Medicine, Kitano Hospital, Osaka, ${ }^{3}$ Department of Internal Medicine, Miyazaki Zenjinkai Hospital, Miyazaki, ${ }^{4}$ Department of Pathology, Graduate School of Medicine, Kyoto University, Kyoto and ${ }^{5}$ Department of Surgical Pathology, Kitano Hospital, Osaka

Received for publication July 9, 2006; Accepted for publication November 1, 2006

Correspondence to Dr. Masataka Matsumoto, ma-matsumoto@kitano-hp.or.jp
} 

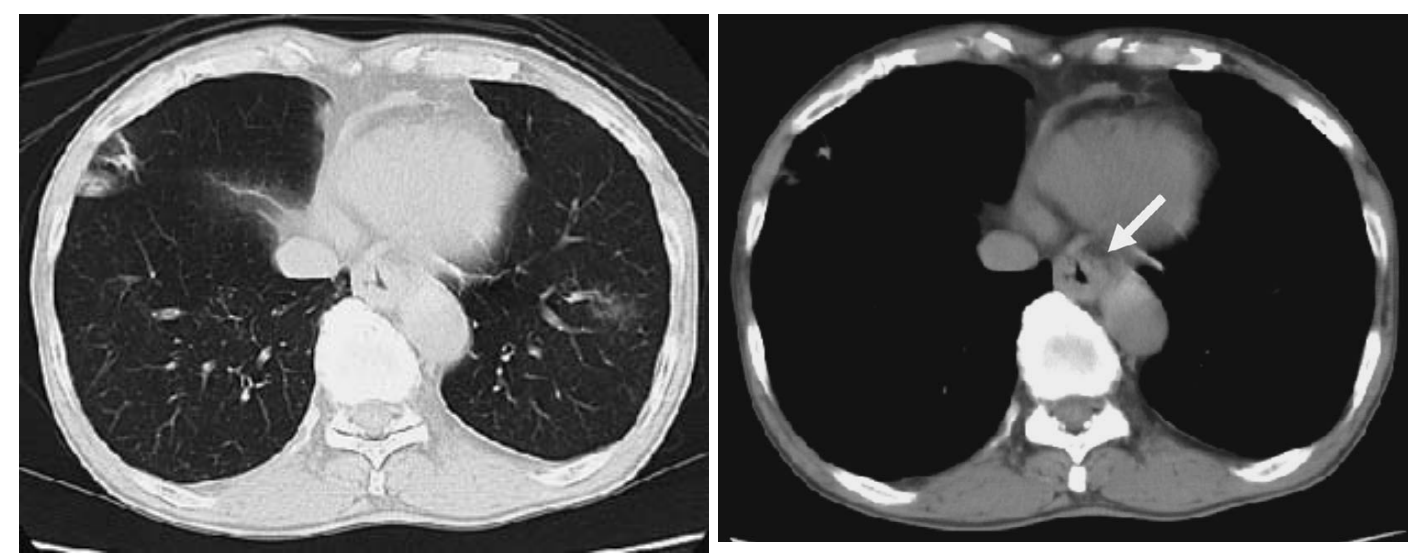

Figure 1. Chest computed tomography in April 2002 showing infiltrative areas and distal esophageal wall thickening (arrow). (A) lung field window setting. (B) mediastinal window setting.
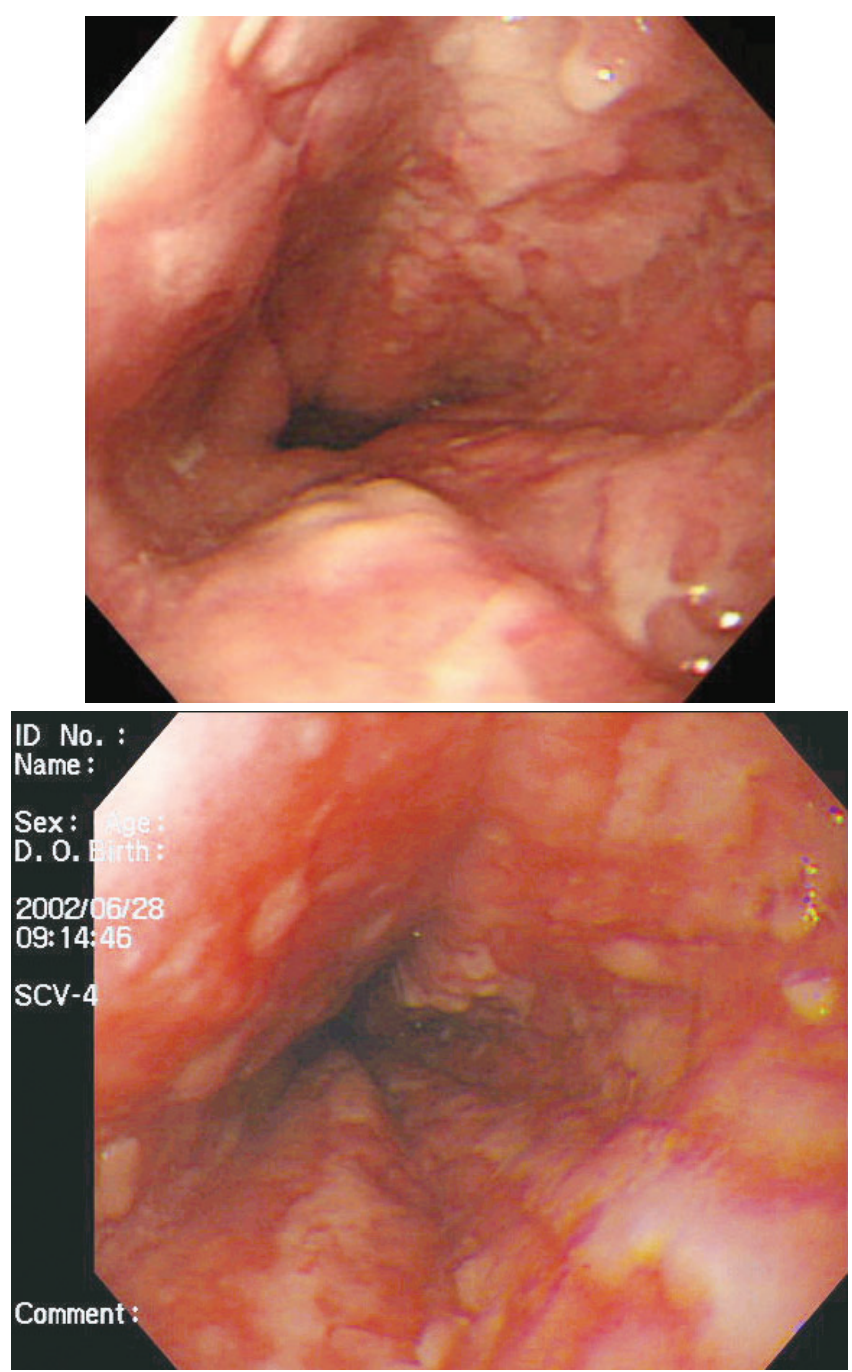

Figure 2. Endoscopic examination in 2002. (A) Endoscopic examination in May 2002 revealed a longitudinal erosion from the middle esophagus to EC junction. (B) Endoscopic examination in June 2002 revealed a worsened longitudinal erosion, which easily bled due to air inflation.

clinically thought that pneumonia was repeatedly caused by meal aspiration refluxed from the stomach, though no chest
CT showed typical aspiration pneumonia until admission in August 2003.

During treatment for pneumonia, we performed endoscopic examination in order to rule out esophageal tumor. Endoscopic examination in May 2002 and June 2002 revealed a longitudinal erosion from the middle esophagus to esophagogastric junction, we clinically diagnosed the lesion as reflux esophagitis (Grade C) (Fig. 2). The pathological finding was severe erosion with inflammation cells consistent with reflux esophagitis. We therefore subscribed proton pump inhibitor (PPI). Dose, duration, and type of PPIs used in this case were lansoprazole $30 \mathrm{mg}$ per day from May 24, 2002 to July 2, 2002, sodium rabeprazole $20 \mathrm{mg}$ per day from July 3, 2002 to October 24, 2002, sodium rabeprazole $10 \mathrm{mg}$ per day from November 27, 2002 to December 17, 2002, lansoprazole $30 \mathrm{mg}$ per day from December 18, 2002 to September 23, 2003, sodium rabeprazole $10 \mathrm{mg}$ per day from September 24, 2003. Though we repeated endoscopy in October 2002 and in January 2003, the esophageal lesion did not show remarkable change.

In April 2003, endoscopy showed irregular ulcer, protrusion and multiple non-dyed areas with Lugol's iodine solution. Histologically we did not find malignant tissue, but did inflammation cell infiltration. The protrusion was histologically diagnosed as granulation (Figs. 3, 4).

In August 2003, he was admitted to the hospital for the third time because of repeated fever for two days, bilateral wrist joint pain and swelling from May 2003 and five kilograms decrease of body weight in a year. He had not taken drugs for joint pain and swelling before the third admission. He was suspected of repeated pneumonia and recurrence of RA. Laboratory data on admission is shown in Table 1 . He also showed symptoms of urine protein, occult blood with red blood cell columns, and positive cytoplasmic antineutrophil cytoplasmic antibody (cANCA).

The head CT showed a tumor in the right maxillary sinus (Fig. 5). Though we performed biopsy twice from the maxillary sinus in September 2003 and October 2003, the pathological findings were not consistent with vasculitis or granu- 

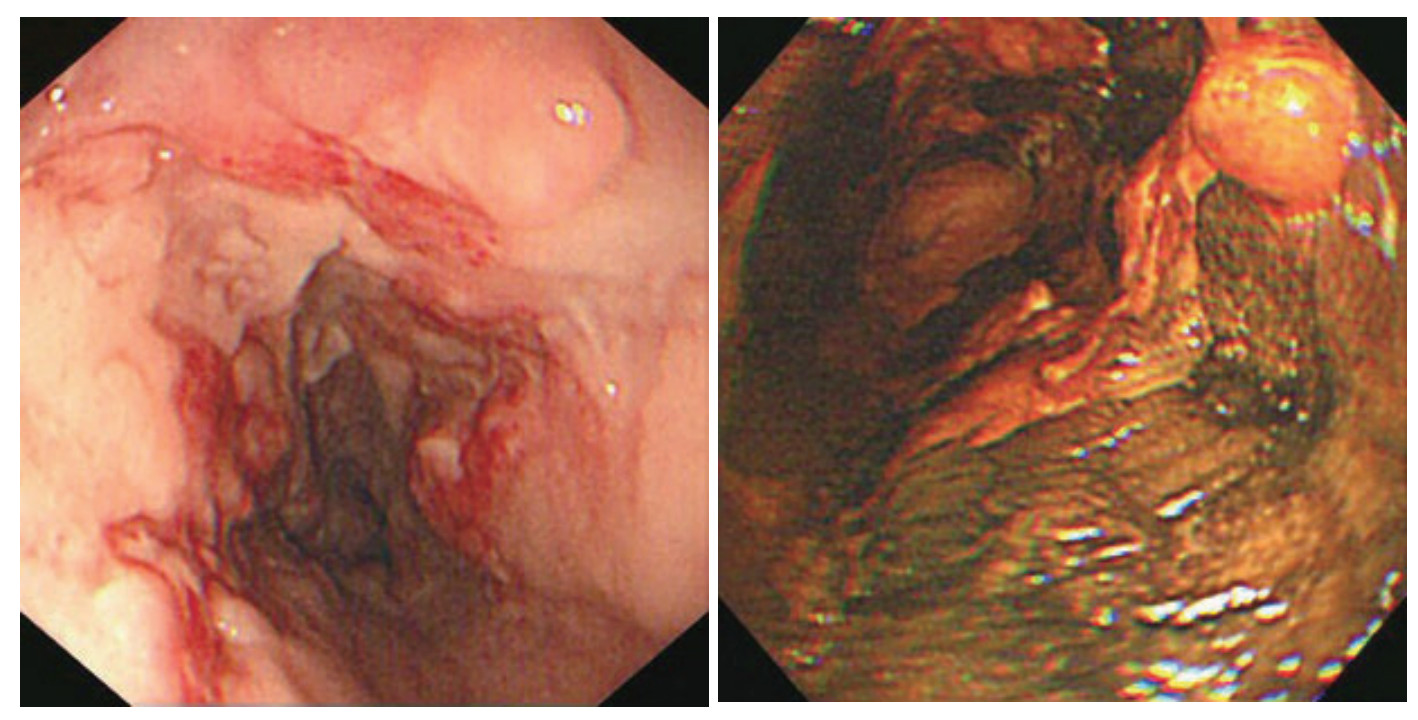

Figure 3. Esophagus before treatment. (A) The endoscope demonstrates erosion, ulcer and protrusion in the middle-distal esophagus. (B) The lesion shows multiple non-dyed areas by Lugol's iodine solution.
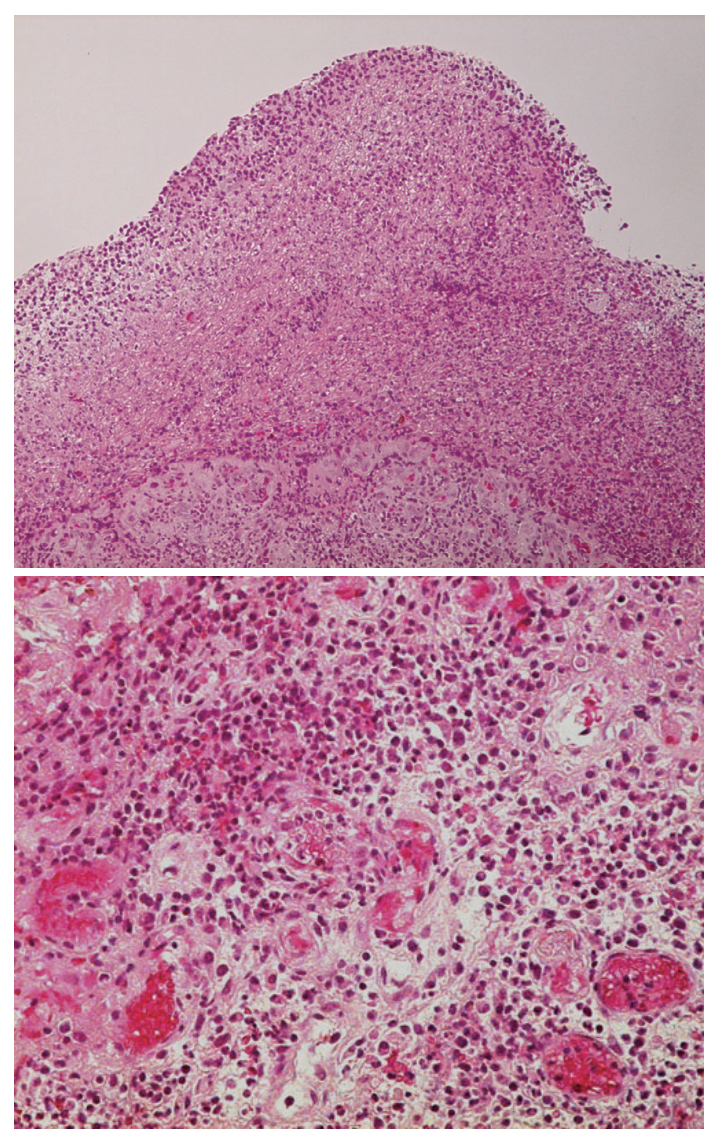

Figure 4. The histological specimen from the esophagus before treatment demonstrates aggregated inflammation cells. HE stain, (A) $\times 100,(B) \times 400$

loma. Based on these signs and symptoms, we diagnosed him as suffering from microscopic polyangiitis (MPA).

To treat him, we used $5 \mathrm{~g}$ of immunoglobulin intravenously for three days. After informed consent, we started

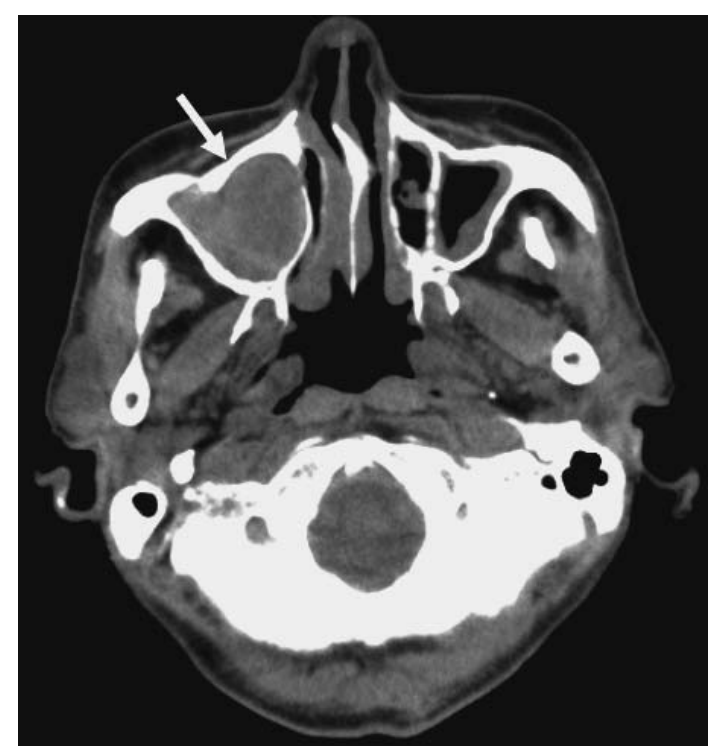

Figure 5. Head CT shows a mass in the right maxillary sinus (arrow), ethmoid bone transformation and mucus membrane thickening in the nasal cavity.

plasma exchange, and gave him $700 \mathrm{mg}$ of cyclophosphamide (CPA) intravenously. His malaise, joint pain and CRP improved markedly. We added $10 \mathrm{mg}$ of oral Prednisolone (PSL) per day, and adopted CPA of 1000-1200 mg per body once a month (Fig. 6). We also used 2 to $3 \mathrm{~g}$ of trimethoprim-sulfamethoxazole (ST) per day. In February 2004, five months after the beginning of medication (PSL, CPA and ST), fever, joint pain, urine findings, cANCA and pneumonia improved remarkably. There was no erosion, ulceration, or protrusion in the lower esophagus on endoscope (Figs. 7, 8). We repeated esophageal endoscopy nine times and took twenty-seven biopsy specimens from May 17, 2002 to August 24, 2004. No biopsy specimens showed vas- 
Table 1. Laboratory Data on Admission in August 2003

\begin{tabular}{|c|c|c|}
\hline \multicolumn{3}{|c|}{ Hematology } \\
\hline WBC & 4800 & $/ \mu 1$ \\
\hline Neut & 49.5 & $\div$ \\
\hline Lym & 33.4 & $\%$ \\
\hline Mo & 7.1 & $1 \%$ \\
\hline Eo & 9.4 & $7 \%$ \\
\hline $\mathrm{Ba}$ & 0.6 & $5 \div$ \\
\hline $\mathrm{Hb}$ & 6.5 & $g / d l$ \\
\hline Ht & 21.2 & $\div$ \\
\hline Ret & 38 & $\%$ \\
\hline Plt & $35.9 \times 10^{4}$ & $/ \mu 1$ \\
\hline \multicolumn{3}{|c|}{ Blood chemistry } \\
\hline AST & 12 & $\mathrm{IU} / \mathrm{I}$ \\
\hline ALT & 10 & $\mathrm{IU} / \mathrm{I}$ \\
\hline $\mathrm{LDH}$ & 386 & $\mathrm{IU} / \mathrm{I}$ \\
\hline ALP & 179 & $\mathrm{IU} / 1$ \\
\hline$\gamma$-GTP & 28 & $8 \mathrm{IU} / 1$ \\
\hline T.Bil & 0.5 & $5 \mathrm{mg} / \mathrm{dl}$ \\
\hline T.pro & 5.5 & $5 \mathrm{~g} / \mathrm{dl}$ \\
\hline Alb & 2.6 & $\mathrm{~g} / \mathrm{dl}$ \\
\hline BS & 92 & $2 \mathrm{mg} / \mathrm{dl}$ \\
\hline T.chol & 123 & $3 \mathrm{mg} / \mathrm{dl}$ \\
\hline TG & 54 & $4 \mathrm{mg} / \mathrm{dl}$ \\
\hline BUN & 13.7 & $7 \mathrm{mg} / \mathrm{dl}$ \\
\hline $\mathrm{UA}$ & 8.8 & $8 \mathrm{mg} / \mathrm{dl}$ \\
\hline $\mathrm{Cr}$ & 0.71 & $1 \mathrm{mg} / \mathrm{dl}$ \\
\hline $\mathrm{Na}$ & 142 & $2 \mathrm{mEq} / \mathrm{l}$ \\
\hline $\mathrm{K}$ & 2.9 & $9 \mathrm{mEq} / \mathrm{l}$ \\
\hline
\end{tabular}

WBC; white blood cell, Weut; neutiophile, Lym; lymphocyte, Mo; monocyte, Eo; eosinophile, $\mathrm{Ba}$; basophile, $\mathrm{Hb}$; hemoglobin, Ht; hematocrit, Ret; reticulocyte, Plt; platelet, AST; aspartate aminotransferase, ALT; alanine aminotransferase, LDH; lactate dehydrogenase, ALP: alkaline phosphatase, $\gamma$-GTP; gamma-glutaryltranspeptidase, T.Bil; total bilirubin, T.pro; total protein, $\mathrm{Alb}$; albmin, BS; blood sugar, T.chol; total cholesterol, TG; triglycerides, BUN; blood urea nitrogen, UA; uric acid, $\mathrm{Cr}$; creatinine, $\mathrm{Na}$; sodium, $\mathrm{K}$; potassium, $\mathrm{Cl}$; chloride, Ca; calcium, CRP; C-reactive protein, $\mathrm{Fe}$; iron, UIBC; unsaturated iron binding capacity, C-ANCA; cytoplasmic antineutrophil cytoplasmic antibodies, P-ANCA; perinulear antineutrophil cytoplasmic antibodies, Anti-GBM Ab; anti-glomerular basement membrane antibody, RF; rheumatoid factor, IgG; immnoglobrin G, C3; complement 3, CH50; complement titer, IC; immune complex, ESR; erythrocyte sedimentation rate

culitis or granuloma.

\section{Discussion}

Wegener's granulomatosis (WG) and MPA are vasculitis syndromes commonly affecting the kidney, and upper and lower respiratory tracts (1). The clinical features of these vasculitis syndromes are very similar and the differential diagnosis is somewhat difficult. At the Chapel Hill Consensus Conference in 1994, WG was defined as necrotizing vasculitis affecting small to medium-sized vessels (e.g., capillaries, venules, arterioles, and arteries) accompanied with granulomatous inflammation involving the respiratory tract. MPA was defined as necrotizing vasculitis with few or no immune deposits, affecting small vessels (i.e., capillaries, venules, arterioles). The name "Wegener's granulomatosis" is restricted to patients with granulomatous inflammation. Patients with

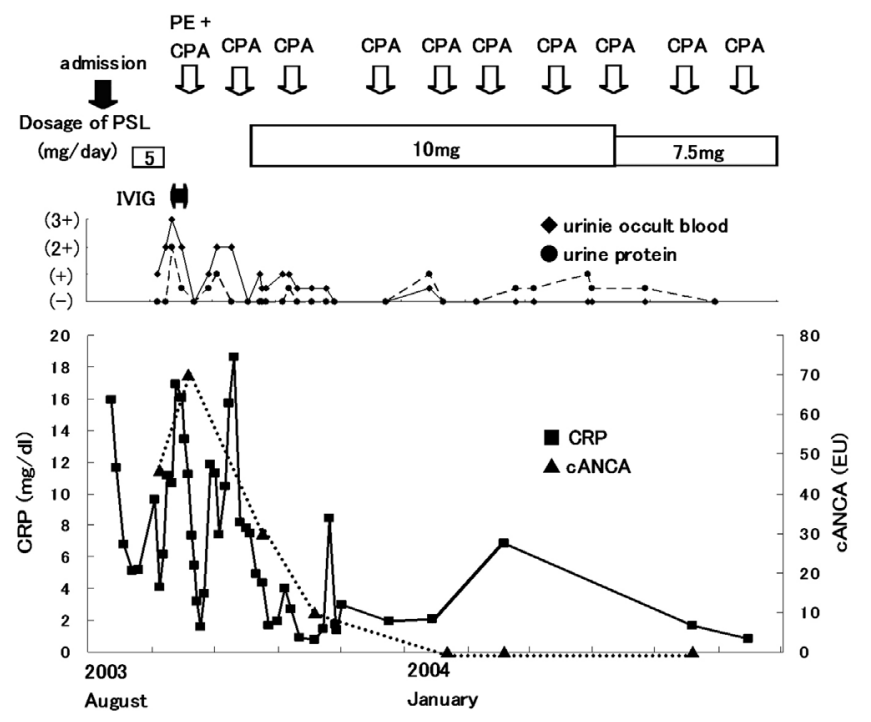

Figure 6. Clinical course after admission. Abbreviations: PE, plasma exchange; CPA, cyclophosphamide; PSL, prednisolone; IVIG, intravenous immunoglobulin; CRP, C-reactive protein; cANCA, cytoplasmic antineutrophil cytoplasmic antibody.

exclusively nongranulomatous small vessel vasculitis involving the upper or lower respiratory tract (e.g., alveolar capillaritis) fall into the category of microscopic polyangiitis (microscopic polyarteritis) (2). In the present case, we did not obtain granuloma histologically, though we sampled biopsy specimens repeatedly both from the esophagus and maxillary sinus. Therefore, in conformity with Chapel Hill Consensus Conference, we diagnosed the patient as MPA.

WG and MPA are commonly referred to as the ANCAassociated vasculitis. The ANCA is antibody to neutrophil cytoplasmic antigen and may constitutively activate primed neutrophils and promote binding of the primed neutrophils to the vascular endothelium, degranulation, and the release of neutrophil chemoattractants, hence creating an autoamplifying loop (1). ANCA is determined by the state of neutrophil activation. cANCA (cytoplasmic ANCA) has been reported to be positive in 70 to $90 \%$ of WG and in approximately $10 \%(5 / 51)$ of MPA, while pANCA (perinuclear ANCA) has been reported to be positive in 50 to $80 \%$ of MPA and in approximately $10 \%$ of WG $(1,3-5)$. In the present case with MPA, cANCA was positive. There is substantial overlap in many of the cases of ANCA-associated vasculitis $(1,6)$.

As the initial treatment of MPA or WG, PSL and CPA are generally used. More than $90 \%$ of patients with WG improve substantially by using PSL and CPA, and there is no apparent difference between MPA and WG in the initial response to treatment $(1,7)$. In the present case, we used PSL and CPA to control MPA $(1,8)$. As a result, symptoms as pneumonia, sinusitis, and esophageal change also, remarkably improved with medical treatment. We, therefore, concluded that the intractable change in the esophagus was one clinical aspect of MPA. In patients with MPA or WG, it is 


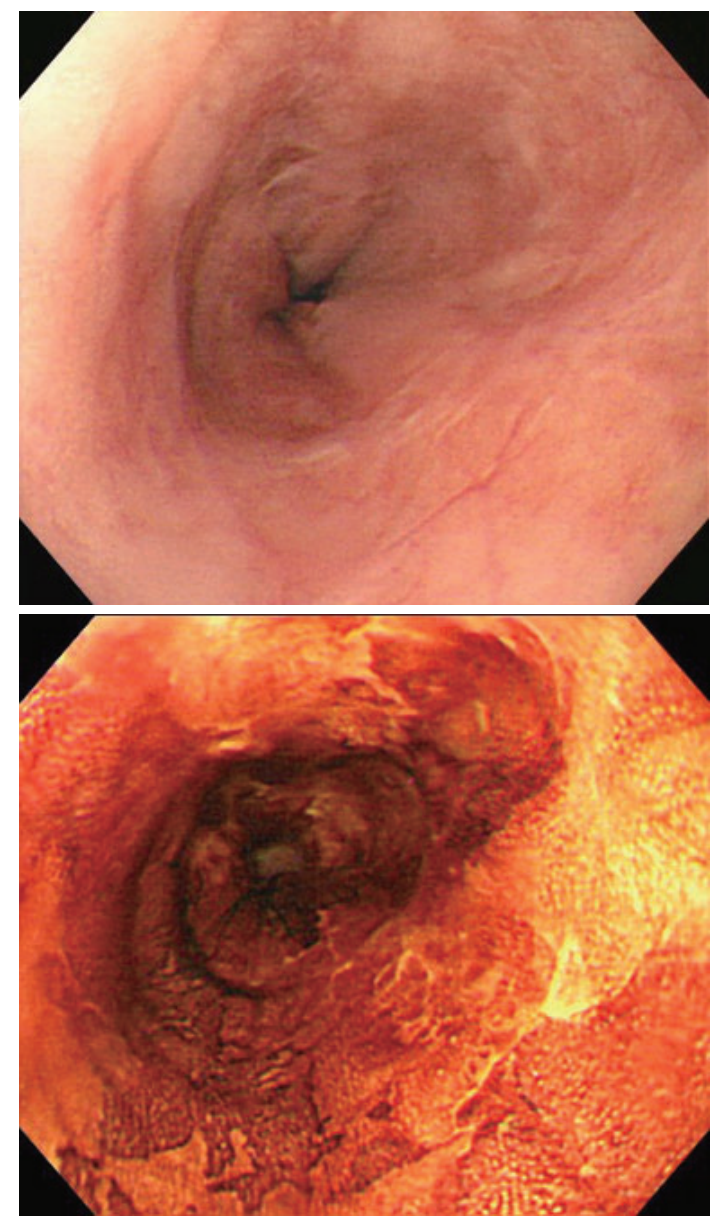

Figure 7. Esophagus after treatment. (A) The endoscope does not show ulcer, erosion or protrusion. (B) The lesion does not show any non-dyed areas by Lugol's iodine solution.

rare to observe lesions in the alimentary tract, compared with those with polyarteritis nodosa or Churg-Strauss syn-

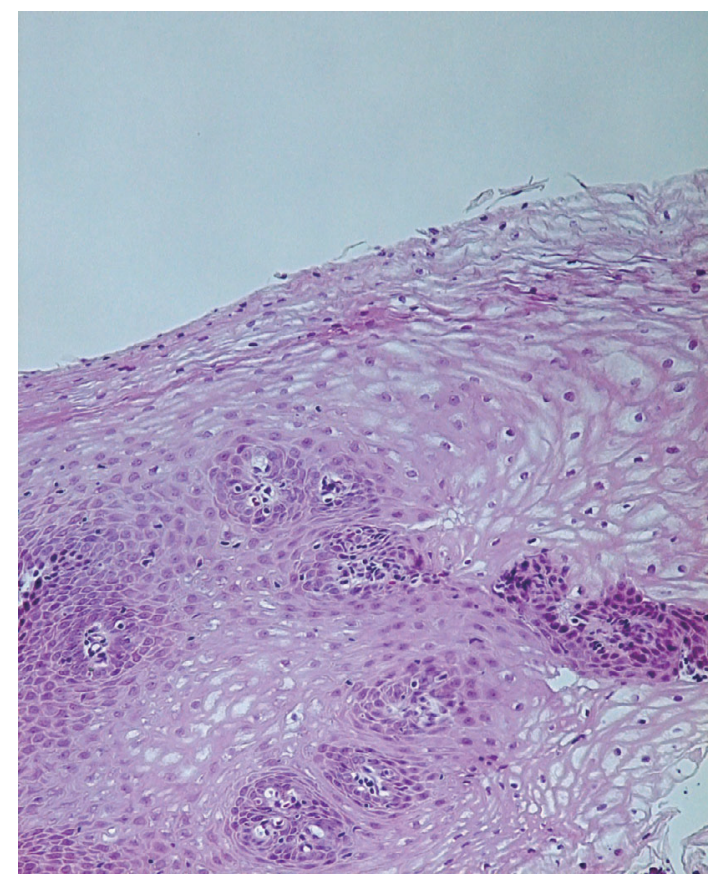

Figure 8. The histological specimen from the esophagus after treatment does not show inflammation cells. HE stain, $\times 400$

drome. In patients with WG, only 4 cases complicated with symptomatic esophageal lesion have been reported (9-12). However, there is no case report of MPA complicated with an esophageal lesion. This is, therefore, the first case report of esophageal involvement in a patient with MPA. In autopsy cases, one out of 29 with WG presented arteritis in the esophagus (13). The lesion in the alimentary tract in patients with WG and MPA may be not rare but uncommon. It may be important to consider vasculitis syndrome when observing intractable esophageal lesions.

\section{References}

1. Seo P, Stone JH. The antineutrophil cytoplasmic antibodyassociated vasculitides. Am J Med 117: 39-50, 2004.

2. Jennette JC, Falk RJ, Andrassy K, et al. Nomenclature of systemic vasculitides. Proposal of an international consensus conference. Arthritis Rheum 37: 187-192, 1994.

3. Langford CA. Wegener Granulomatosis. Am J Med Sci 321: 76$82,2001$.

4. Guillevin L, Durand-Gasselin B, Cevallos R, et al. Microscopic polyangiitis: clinical and laboratory findings in eighty-five patients. Arthritis Rheum 42: 421-430, 1999.

5. Hashimoto H. Microscopic polyangiitis. Nippon Rinsho 63 Suppl 5: 330-336, 2005 (in Japanese).

6. Falk RJ, Jennette JC. Thoughts about the classification of small vessel vasculitis. J Nephrol 17 Suppl 8: S3-9, 2004.

7. Smyth L, Gaskin G, Pusey CD. Microscopic polyangiitis. Semin Respir Crit Care Med 25: 523-533, 2004.

8. Jennette JC, Falk RJ. Antineutrophil cytoplasmic autoantibodies and associated diseases: a review. Am J Kidney Dis 15: 517-529,
1990.

9. Spiera RF, Filippa DA, Bains MS, Paget SA. Esophageal involvement in Wegener's granulomatosis. Arthritis Rheum 37: 14041407, 1994.

10. Featherstone RJ, Camero LG, Khatib R, Snower D, Mungara P. Massive esophageal bleeding in achalasia complicated by cytomegalovirus esophagitis. Ann Thorac Surg 59: 1021-1022, 1995.

11. Fallows GA, Hamilton SF, Taylor DS, Reddy SB. Esophageal involvement in Wegener's granulomatosis: a case report and review of the literature. Can J Gastroenterol 14: 449-451, 2000.

12. Miller PG, Santini C, Freed MJ. Dysphagia in a patient with Wegener's granulomatosis: case report. Dysphagia 16: 136-139, 2001.

13. Fahey JL, Leonard E, Churg J, Godman G. Wegener's granulomatosis. Am J Med 17: 168-179, 1954.

(C) 2007 The Japanese Society of Internal Medicine http://www.naika.or.jp/imindex.html 